\title{
Synchronous Cervical Minimal Deviation Adenocarcinoma, Gastric Type Adenocarcinoma and Lobular Endocervical Glandular Hyperplasia Along with STIL in Peutz-Jeghers Syndrome: Eliciting Oncogenesis Pathways
}

\author{
Azfar NEYAZ' ${ }^{1}$, Nuzhat HUSAIN' ${ }^{\text {, Manish DEODHAR }}{ }^{2}$, Rohini KHURANA ${ }^{3}$, Saumya SHUKLA ${ }^{1}$, Aditi ARORA ${ }^{1}$ \\ Department of ${ }^{1}$ Pathology, ${ }^{2}$ Radiology and ${ }^{3}$ Radiation Oncology, Dr Ram Manohar Lohia Institute of Medical Sciences, LUCKNOW, INDIA
}

\begin{abstract}
We describe an unusual case of a Peutz-Jeghers syndrome associated with a composite synchronous cervical neoplasia comprising precursor "lobular endocervical glandular hyperplasia (LEGH)", "minimal deviation adenocarcinoma (MDA)" and "gastric-type adenocarcinoma (GTA)" along with a serous tubal intraepithelial lesion (STIL) in the right fallopian tube. A 24-year-old woman presented with a white mucoid discharge and bleeding per vaginum for one year. Histopathological evaluation showed MDA \& GTA in FIGO grade III with pelvic lymph node metastasis despite a deceptively bland tumour morphology and low Ki-67 index, indicating an aggressive tumour course and poor prognosis. Diagnostic marker profile in the cervix showed gastric type mucin and positive expression of CK-7, CK-20 (patchy), CEA, and negative CDX-2, p16, ER and PR. Further an attempt at eliciting the oncogenesis pathway in view of the p16 and HPV negative nature of the gastric type cervical adenocarcinoma showed negativity for $\mathrm{p} 53$ but activation of cyclin D1. Growth factors including Her2 and EGFR were negative while VEGFR was over-expressed. She was treated by radical hysterectomy and pelvic radiation. She was free from recurrence at the 12-month follow-up. This is a first-time report of a STIL in the fallopian tube which was validated by a unilateral mutant type p53 expression and increased Ki67 index, associated with synchronous gastric type adenocarcinoma of the cervix in all stages of evolution.
\end{abstract}

Key Words: Peutz-Jeghers syndrome, Minimal deviation adenocarcinoma, Uterine cervical neoplasm, Gastric type adenocarcinoma, Lobular endocervical glandular hyperplasia

\section{INTRODUCTION}

Peutz-Jeghers syndrome (PJS) is an autosomal dominant inherited disorder that is characterized by hamartomatous polyps of the gastrointestinal tract and mucocutaneous melanin pigmentation and is often accompanied with a higher incidence of other malignancies, such as breast cancer, adenoma malignum of the uterine cervix and sex cord tumor with annular tubules in the ovary. The cumulative cancer risk for a PJS patient is $85 \%$ by age 70 (control population risk 18\%) (1). Serous tubal intraepithelial lesion (STIL) in the fallopian tube has not been described in PJS in the literature. We describe a very unusual case of PJS with a right unilateral STIL in the fallopian tube associated with a composite synchronous cervical malignancy comprising a range of mucinous lesions of the uterine cervix from precursor lobular endocervical glandular hyperplasia (LEGH), minimal deviation adenocarcinoma (MDA) to gastric-type adenocarcinoma (GTA).

(Turk Patoloji Derg 2019, 35:247-253)

Received : 30.03.2017 Accepted : 14.06.2017

\section{CASE REPORT}

A 24-year old G1P0 woman presented with white mucoid discharge and bleeding per vaginum for one year. Her past medical history was unremarkable. On investigation, USG abdomen revealed a multicystic mass replacing the uterine cervix. Pelvic magnetic resonance imaging (MRI) revealed expansion of the cervix with a multilocular cystic mass having thin septae appearing hypointense on T1WI and hyperintense on T2WI and containing a fluid-fluid level due to dependent layering (Figure 1A-D).

The first excisional biopsy was reported as mucinous adenocarcinoma. Subsequently, the patient underwent radical hysterectomy with bilateral salpingo-oophorectomy, vaginal reconstruction and bilateral pelvic lymph node dissection.

On macroscopic examination, the cervix was infiltrated by an ill-defined ulcero-infiltrative growth measuring $4.0 \times 3.0 \times 3.0 \mathrm{~cm}$ and involving the endocervix, ectocervix

Correspondence: Azfar NEYAZ

Dr Ram Manohar Lohia Institute of Medical Sciences,

Department of Pathology, LUCKNOW, INDIA

E-mail: azfarneyaz@gmail.com Phone: +91 9557340803 
and isthmus. The tumour was seen to be infiltrating the entire thickness of the cervix. The ovaries, fallopian tubes and parametrium appeared free in gross evaluation. Macroscopic metastases to pelvic lymph nodes were evident. The cervix was extensively sectioned. Sections from bilateral fallopian tubes were also taken as per the Sectioning and Extensively Examining of the Fimbriated end (SEE-FIM) protocol (2). Microscopically, complete effacement of cervical stroma by three distinct histological patterns was noted. The majority of the tumor was composed of dilated irregularly shaped glands lined by single-layer of columnar cells without atypia. These well differentiated glands lined by columnar cells displayed basally located bland nuclei along with abundant intracytoplasmic mucin.
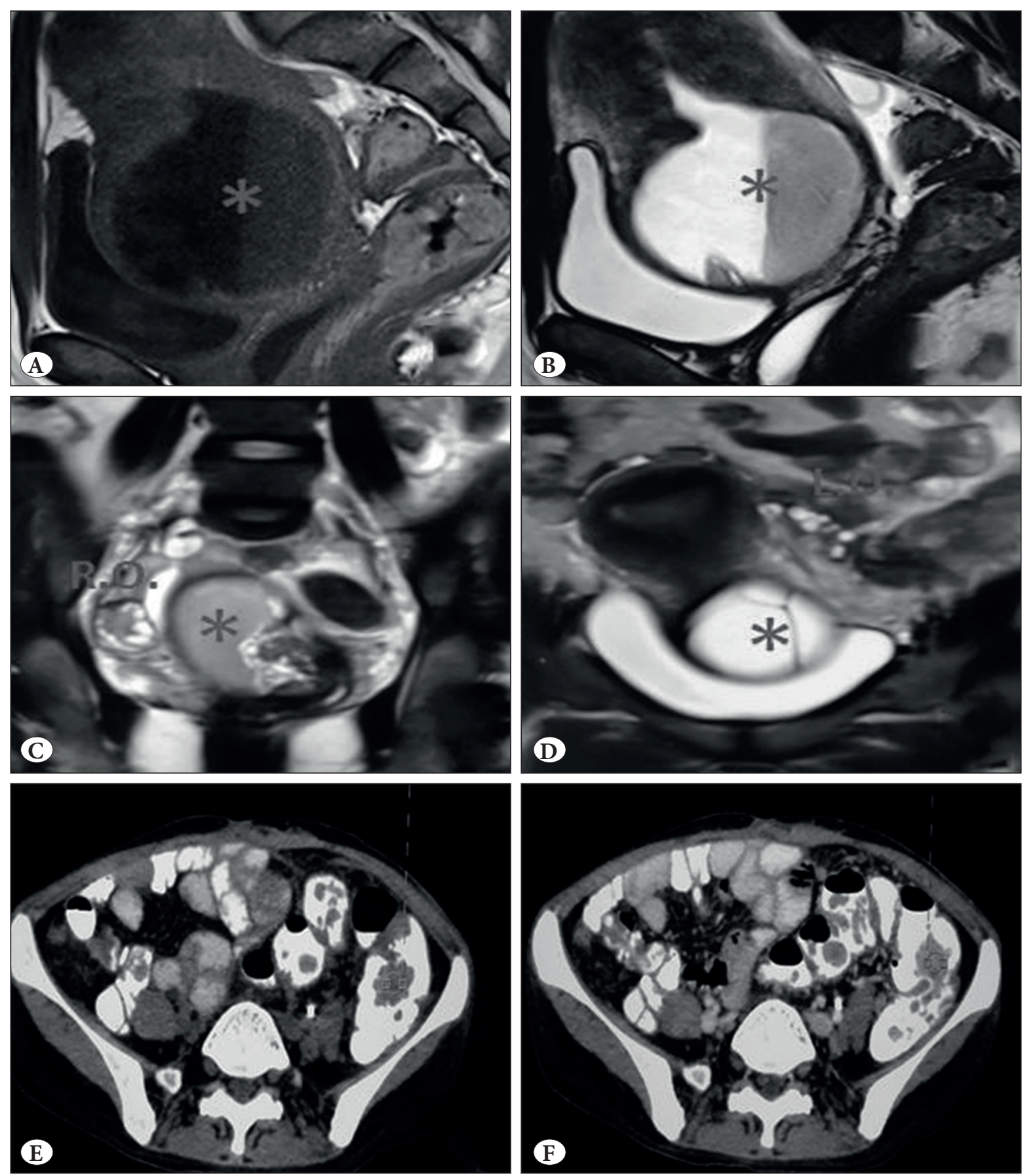

Figure 1: Preoperative pelvic MRI scan shows A) Sagittal T1WI and B) T2WI demonstrating a complex cystic mass $\left({ }^{\star}\right)$ containing fluid-fluid levels involving the cervix. C-D) Coronal T2WI demonstrates the ovaries separate from the cystic mass. E) Non-contrast and F) Contrast enhanced follow-up CT scans performed with bowel opacification show multiple enhancing bowel polyps. 
Mitosis, atypia or necrosis was not apparent. Some of the glands showed complex branching and distortion. These microscopic findings were consistent with a diagnosis of minimal deviation adenocarcinoma (Figure 2A-C). Encroachment of the blood vessels and nerve fibers added further support for stromal invasion. The other histological pattern in spatial continuity with the first type constituted irregularly shaped glands revealing moderate atypia and stromal desmoplasia. These ill-defined neoplastic glands showed moderate anisonucleosis with nuclear stratification, coarse chromatin, occasional prominent nucleoli and large to abundant amount of eosinophillic to clear cytoplasm reflecting morphological features of gastric type mucinous adenocarcinoma of the cervix (Figure 2D-F). Cytochemical staining highlighted neutral mucin (Figure 2G). Accompanying lobular endocervical glandular hyperplasia consisting of proliferating moderately sized endocervical glands confined within the superficial cervical stroma was discernible at places (Figure $2 \mathrm{H}$ ). Synchronous existence of three diverse histological morphologies prompted the diagnosis of composite cervical adenocarcinoma composed of minimal deviation of adenocarcinoma and gastric type adenocarcinoma. The tumour involved full thickness of the cervix lip. Foci of perineural invasion and lympho-vascular invasion were also observed. Tumour was seen extending to lower uterine segment and anterior vaginal wall. Bilateral
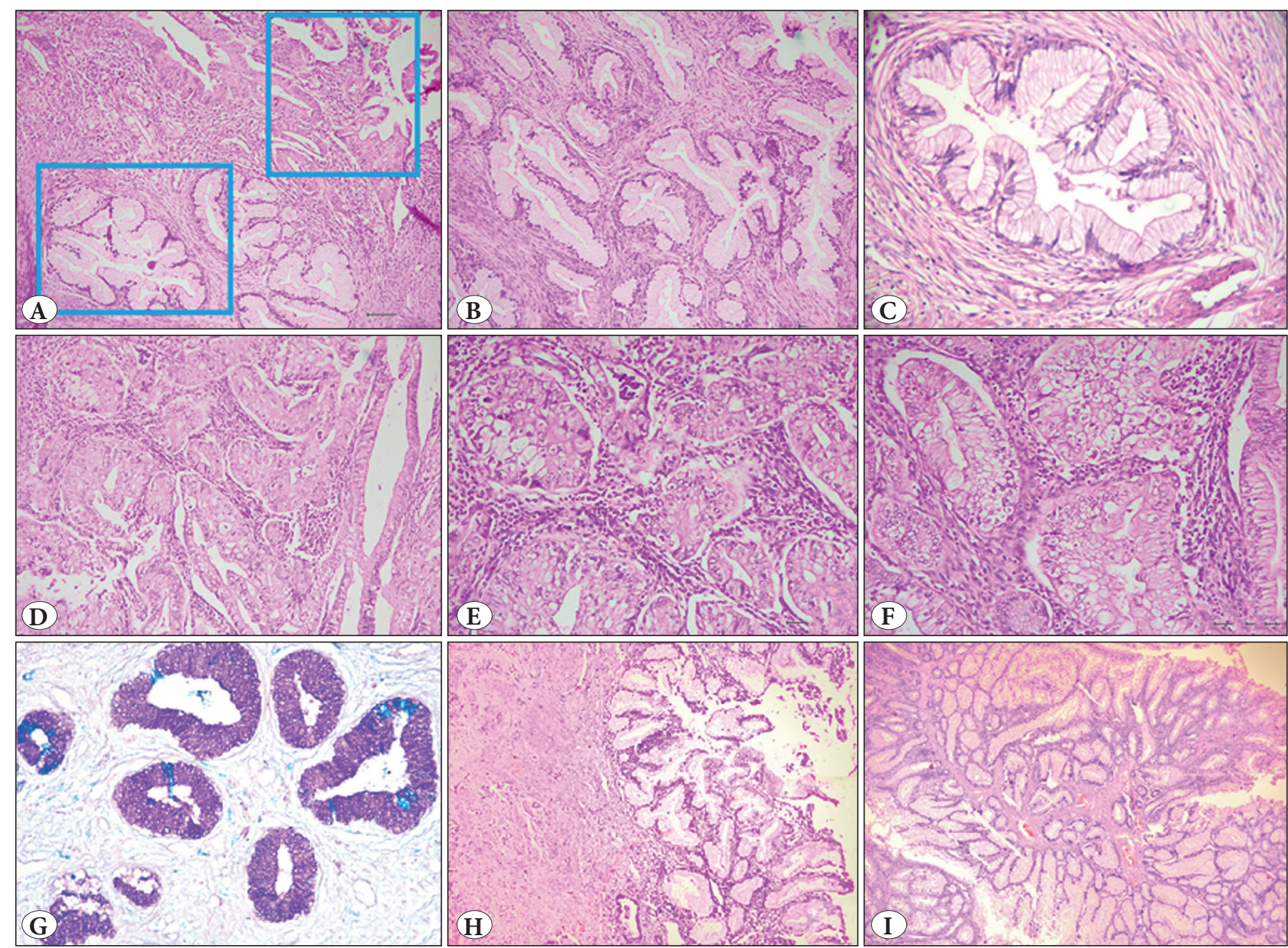

Figure 2: A) Two histologically distinct components, MDA and GTA identified (H\&E; x100). B) Irregular neoplastic glands displaying complex branching (H\&E; x100). C) Tumour glands lined by single layer of columnar cells with basally located bland nuclei, suggesting MDA (H\&E; x400). D-F) Irregularly shaped dilated glands with moderate atypia and prominent infoldings and neoplastic glands with nuclear pseudostratification, enlarged centrally placed nuclei, prominent nucleoli and abundant foamy cytoplasm, features suggestive of GTA (H\&E; x100; x100;x200). G) Combined Alcian blue \& PAS-D highlights the neutral mucin (Alcian blue\&PAS-D; x200). H) Lobular endocervical glandular hyperplasia consisting of proliferating moderately sized endocervical glands (H\&E; x40). I) Hamartomatous GI polyp composed of complex arborizing glandular architecture lying on slips of smooth muscle (H\&E; x40). 
pelvic lymph nodes showed metastasis and hence, was staged as FIGO IIIA. Serous tubal intraepithelial lesion (STIL) was noted in right fallopian tube, characterised by non-ciliated epithelium displaying nuclear enlargement, hyperchromasia, irregularly distributed chromatin and nucleolar prominence (Figure 3A-B). Strong p53 overexpression was evident (Figure 3C) with Ki-67 index of approximately $8 \%$ (Figure 3D). The lesion was evident in the distal half of the tube and at the fimbrial end. Other tube showed no morphological atypia with wild type p53, low Ki67. The ovaries did not show any neoplastic pathology.

Immunohistochemical evaluation of the cervical adenocarcinoma and the metastasis in the lymph nodes was carried out to study the expression of recognised markers such as CK-7, CK-20, CDX-2, CEA, p16, p53,
ER, PR and Ki-67 as well as Her 2, Cyclin D1, EGFR, VEGFR and $\beta$-catenin to elicit pathways of carcinogenesis. Strong or diffuse immunoreactivity was observed for CK7, CEA, Cyclin D1 and VEGFR while CDX-2, p16, p53, Her-2, EGFR, ER and PR were negative (Figure 4 A-L). CK-20 showed focal expression. Ki-67 in the MDA, GTA component and lymph node metastasis was 3-4\%. Uniform marker expression was observed in the primary lesion and the lymph node metastasis, and in the latter the phenotype was predominantly MDA.

Follow-up imaging by CT scan showed no residual mass in the pelvis. However, numerous polyps were incidentally detected in the small and large intestines. An upper and lower gastrointestinal endoscopy was done, disclosing multiple polyps throughout the duodenum, colon and
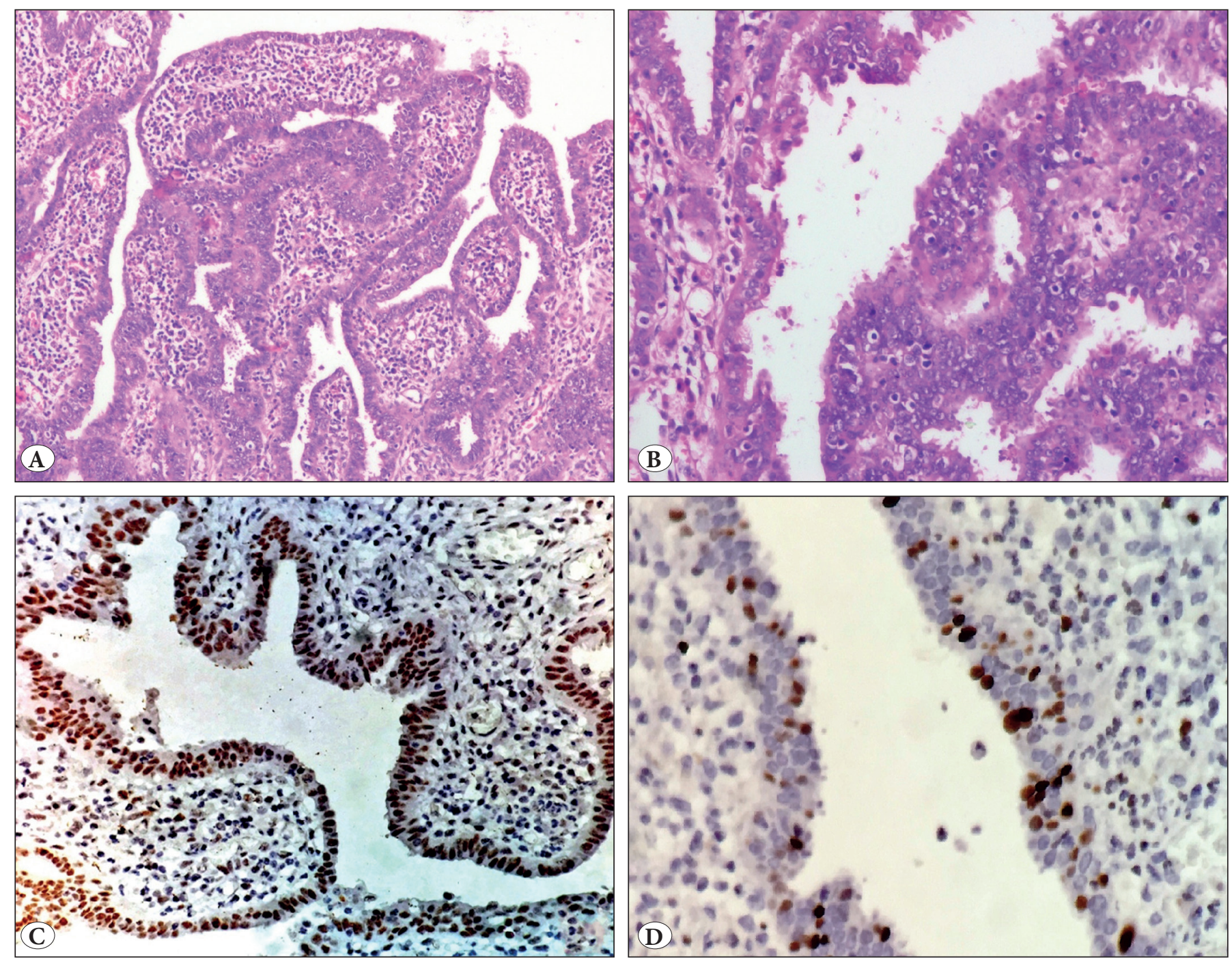

Figure 3: A-B) Serous tubal intraepithelial lesion (STIL) characterised by stratified epithelium displaying increased nuclear/cytoplasmic ratio, nuclear atypia (H\&E; x200; x400). C) Mutant type p53 over-expression in STIL (p53; x200). D) Ki-67 index at 8\% in STIL lesion (Ki-67; x400). 
rectum (Figure 1E,F). Polypectomy was done from the largest rectal polyp. Histological examination exhibited complex arborizing glandular architecture lying on slips of smooth muscle supporting aggregates of benign cystically dilated glands suggestive of hamartomatous polyp (Figure 2I). Features of concomitant dysplasia or malignancy were not seen.

Clinical examination disclosed mucocutaneous pigmentation in the peri-oral area. However, no significant family history could be elicited. The patient was treated by radical hysterectomy and pelvic radiation on a LINAC at 45 gy in 25 sittings of radiation followed by brachytherapy to the vault. She was free from recurrence during the 12 month follow-up.

\section{DISCUSSION}

We report an interesting rare composite lesion in the uterine cervix with a morphologic spectrum of LEGH and malignant cervical mucinous lesions exhibiting gastric differentiation including MDA and GTA existing in synchronous continuous transition in a case of PeutzJeghers syndrome. We are reporting for the first time an associated STIL in fallopian tube in PJS. In an extensive literature review, we have not come across any reports of serous intraepithelial lesions of the tube associated with PJS. Rare reports of mucinous lesions of the fallopian tube have been reported in association with other mucinous lesions of the female genital tract in GTA, MDA and with Peutz-Jeghers syndrome (3). It was interesting to note that STIL was unilaterally present in right fallopian tube. The diagnosis was validated using a diagnostic algorithm recommended by Vang et al. coordinating the histology and immunohistochemical expression of p53 and the Ki67 index (4). STIL and STIC are known to be associated with serous carcinomas of peritoneal, ovarian, or endometrial origin and not with any other non-serous lesions such as endometrioid, clear cell, or mucinous carcinomas (5). In cases with concordant STICs and ovarian or pelvic high grade serous carcinomas, identical TP53 mutation in both STICs and the associated ovarian neoplasms has been demonstrated (6), however it is significant to note in our case that the cervical malignancy was p53 negative while STIL was p53 positive denoting multifocal unrelated neoplastic change in association with PJS.
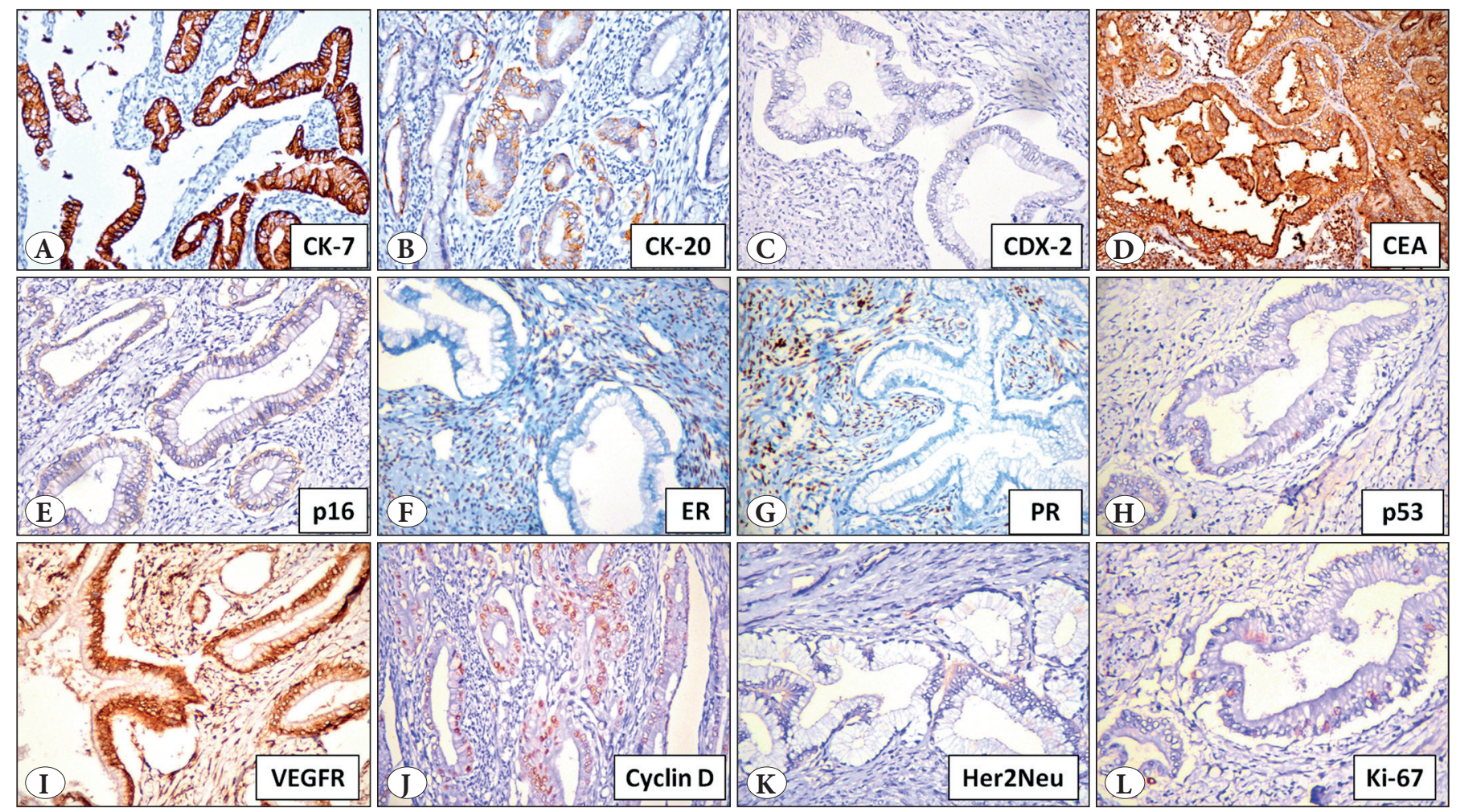

Figure 4: A) Positive immunoreactivity for CK-7 (CK-7; x100). B) Focal positivity for CK-20 (IHC; x100). C) Negative immunoreactivity for CDX-2 (IHC; x200). D) Positive immunoreactivity for CEA (IHC; x100). E) Negative immunoreactivity for p16 (IHC; x100). F) Negative immunoreactivity for ER (IHC; x200). G) Negative immunoreactivity for PR (IHC; x200). H) Negative immunoreactivity for p53 (IHC; x200). I) Positive immunoreactivity for VEGFR (IHC; x100). J) Positive immunoreactivity for Cyclin D1 (IHC; x100). K) Negative immunoreactivity for Her-2 (IHC; 200). L) Ki-67 index was 3-4\% (IHC; x200). 
The term 'lobular endocervical glandular hyperplasia' (LEGH) has been shown to have a gastric-type IHC profile and is considered to be a putative precursor lesion to MDA and GTA. Approximately 10-15\% of PJS cases develop minimum deviation adenocarcinoma. Rare case reports describe the presence of LEGH, a potential premalignant lesion of MDA, and its evolution to more aggressive gastric adenocarcinoma (7). McCluggage et al. reported composite carcinoma for the first time and identified common genetic aberrations between MDA and GTA indicating a possible link between these tumors. In their case, the MDA and GTA occupied discrete areas in the cervix (8). In contrast, the MDA and GTA components in the current case as well as the case reported by Peng et al. were continuous in nature, and had similar immunophenotypes, favoring the hypothesis that GTA may arise from dedifferentiation of MDA (9). MDA has been classified as an extremely well differentiated form of GTA in the new WHO Classification of Tumors of Female Reproductive Organs. Although P53 postitivty has been enumerated as one of the diagnostic criteria for the tumour, Carleton et al. have shown "wildtype" p53 in 58\% and "mutation-type" staining in $41 \%$ of the cases (10). In the current case, p53 was negative while cyclin D1 and VEGFR were strongly expressed in the tumour nuclei and membrane respectively.

High-risk human papillomavirus (hr-HPV) is currently considered to be implicated in the carcinogenesis of most cervical adenocarcinoma and squamous cell carcinoma with a detection rate reaching $85 \%$ in the former. Unlike conventional endocervical adenocarcinoma, the presence of hr HPV and diffuse $\mathrm{p} 16$ positivity, a surrogate marker for HPV integration, is not associated with MDA and gastric type adenocarcinoma. Mucinous cervical adenocarcinomas appear to represent an oncogenic hr-HPV-independent pathway of p16 overexpression and are therefore a potential pitfall of HPV DNA testing and vaccination (11). Our case was also negative for p16 expression.

Immmunohistochemical support for a gastric phenotype comes from studies demonstrating histochemical positivity for gastric mucin and immunohistochemical positivity for the gastric markers HIK1083 and MUC6. Histological changes mimicking clear cell carcinoma have been reported after neoadjuvant therapy but immunohistochemical positive expression of CEA, cytokeratin 7 (diffuse), and cytokeratin 20 (focal), positive HIK1083 stain or negative/focal p16 stain favors gastric-type mucinous adenocarcinoma of the cervix (12). Immunophenotypic overlap with pancreaticobiliary adenocarcinomas exists but PAX8 immunoreactivity may be useful to distinguish them.

The responsible gene in PJS is a tumor suppressor gene, STK11/LKB1, that islocated on the shortarm of chromosome 19 (19p13.3). STK11/LKB1 is a $23-\mathrm{kb}$ gene that encodes a serine-threonine kinase of a 432 -amino-acid protein and consists of 9 coding exons and 1 non-coding exon (13). Cases with MDA in PJS, that is a STK11 mutation, have a poorer prognosis than those without a STK11 mutation $(\mathrm{p}=0.039)$ (14). There are no established guidelines for screening for gynecologic neoplasia in PJS patients (15). Routine cervical cytology screening is recommended and ideally the diagnosis of PJS should be communicated to the reviewing cytotechnologist/cytopathologist so that careful assessment for gastric type adenocarcinoma is done. Beyond cervical cytology screening, some investigators advocate for yearly pelvic examinations and/or ultrasound beginning in young adulthood to detect ovarian neoplasia; however, this recommendation has not been uniformly accepted $(15,16)$.

The treatment schedule for MDA is the same as that for conventional adenocarcinoma at the same stage. Surgery remains the main treatment for patients with early stage lesions. Radiotherapy and/or chemotherapy are recommended for patients with advanced disease. Our case underwent a radical hysterectomy followed by radiation therapy.

Gastric-type adenocarcinoma and MDA have been reported to have an aggressive clinical course. Carleton $\mathrm{C}$ et al. reported in their review of 40 cases of GTA that 59\% were in the advanced stage (FIGO II-IV), 50\% had lymph node metastases, $35 \%$ had ovarian involvement, $20 \%$ had abdominal disease, $39 \%$ had at least one site of metastasis at the time of initial surgery and $12 \%$ of the patients experienced distant recurrence (10). The 5-year disease-free survival rate of patients with gastric-type adenocarcinoma (38\%) was substantially lower than that of patients with the usual type of uterine cervical adenocarcinoma (74\%). In the study by Kojima et al., gastric morphology and immunophenotype (HIK1083-positivity) were found to be independent predictive factors of disease recurrence and decreased survival in stage I and II cervical adenocarcinoma (17). The minimal deviation adenocarcinoma also has a less favorable prognosis than the usual type of endocervical adenocarcinoma. The 2-year survival rate of patients with any stage of minimal deviation adenocarcinoma is $20-30 \%$, whereas that of patients with stage I disease is around $50 \%$ (18). 


\section{REFERENCES}

1. Hearle N, Schumacher V, Menko FH, Olschwang S, Boardman LA, Gille JJ, Keller JJ, Westerman AM, Scott RJ, Lim W, Trimbath JD, Giardiello FM, Gruber SB,Offerhaus GJ, de Rooij FW, Wilson JH, Hansmann A, Möslein G, Royer-Pokora B,Vogel T, Phillips RK, Spigelman AD, Houlston RS. Frequency and spectrum of cancers in the Peutz-Jeghers syndrome. Clin Cancer Res. 2006;12:3209-15.

2. Mingels MJ, van Ham MA, de Kievit IM, Snijders MP, van Tilborg AA, Bulten J, Massuger LF. Müllerian precursor lesions in serous ovarian cancer patients: Using the SEE-Fim and SEE-End protocol. Mod Pathol. 2014;27:1002-13.

3. Seidman JD. Mucinous lesions of the fallopian tube. A report of seven cases. Am J Surg Pathol. 1994;18:1205-12.

4. Vang R, Visvanathan K, Gross A, Maambo E, Gupta M, Kuhn E, Li RF, Ronnett BM, Seidman JD, Yemelyanova A, Shih IeM, Shaw PA, Soslow RA, Kurman RJ. Validation of an algorithm for the diagnosis of serous tubal intraepithelial carcinoma. Int J Gynecol Pathol. 2012;31:243-53.

5. Tang S, Onuma K, Deb P, Wang E, Lytwyn A, Sur M, Daya D. Frequency of serous tubal intraepithelial carcinoma in various gynecologic malignancies: A study of 300 consecutive cases. Int J Gynecol Pathol. 2012;31:103-10.

6. Kuhn E, Kurman RJ, Vang R, Sehdev AS, Han G, Soslow R, Wang TL, Shih IeM. TP53 mutations in serous tubal intraepithelial carcinoma and concurrent pelvic high-grade serous carcinoma-evidence supporting the clonal relationship of the two lesions. J Pathol. 2012;226:421-6.

7. Kawauchi S, Kusuda T, Liu XP, Suehiro Y, Kaku T, Mikami Y, Takeshita M, Nakao M, Chochi Y, Sasaki K. Is lobular endocervical glandular hyperplasia a cancerous precursor of minimal deviation adenocarcinoma?: A comparative moleculargenetic and immunohistochemical study. Am J Surg Pathol. 2008;32:1807-15.

8. McCluggage WG, Harley I, Houghton JP, Geyer FC, MacKay A, Reis-Filho JS. Composite cervical adenocarcinoma composed of adenoma malignum and gastric type adenocarcinoma (dedifferentiated adenoma malignum) in a patient with Peutz Jeghers syndrome. J Clin Pathol. 2010;63:935-41.

9. Peng WX, Kure S, Ishino K, Kurose K, Yoneyama K, Wada R, Naito Z. P16-positive continuous minimal deviation adenocarcinoma and gastric type adenocarcinoma in a patient with Peutz-Jeghers syndrome. Int J Clin Exp Pathol. 2015;8:5877-82.
10. Carleton C, Hoang L, Sah S, Kiyokawa T, Karamurzin YS, Talia KL, Park KJ, McCluggage WG. A detailed immunohistochemical analysis of a large series of cervical and vaginal gastric-type adenocarcinomas. Am J Surg Pathol. 2016;40:636-44.

11. Park KJ, Kiyokawa T, Soslow RA, Lamb CA, Oliva E, Zivanovic O, Juretzka MM, Pirog EC. Unusual endocervical adenocarcinomas: An immunohistochemical analysis with molecular detection of human papillomavirus. Am J Surg Pathol. 2011;35:633-46.

12. Mikami Y, Kiyokawa T, Hata S, Fujiwara K, Moriya T, Sasano H, Manabe T, Akahira J, Ito K, Tase T, Yaegashi N, Sato I, Tateno H, Naganuma H. Gastrointestinal immunophenotype in adenocarcinomas of the uterine cervix and related glandular lesions: A possible link between lobular endocervical glandular hyperplasia/pyloric gland metaplasia and 'adenoma malignum'. Mod Pathol. 2004;17:962-72.

13. Hemminki A, Tomlinson I, Markie D, Järvinen H, Sistonen $P$, Björkqvist AM, Knuutila S, Salovaara R, Bodmer W, Shibata D, de la Chapelle A, Aaltonen LA. Localization of a susceptibility locus for Peutz-Jeghers syndrome to $19 \mathrm{p}$ using comparative genomic hybridization and targeted linkage analysis. Nat Genet. 1997;15:87-90.

14. Kuragaki C, Enomoto T, Ueno Y, Sun H, Fujita M, Nakashima R, Ueda Y, Wada H, Murata Y, Toki T, Konishi I, Fujii S. Mutations in the STK11 gene characterize minimal deviation adenocarcinoma of the uterine cervix. Lab Invest. 2003;83:35-45.

15. Meserve EE, Nucci MR. Peutz-Jeghers Syndrome: Pathobiology, pathologic manifestations, and suggestions for recommending genetic testing in pathology reports. Surg Pathol Clin. 2016;9:24368.

16. McGarrity TJ, Kulin HE, Zaino RJ. Peutz-Jeghers syndrome. Am J Gastroenterol 2000;95:596-604.

17. Kojima A, Mikami Y, Sudo T, Yamaguchi S, Kusanagi Y, Ito M, Nishimura R. Gastric morphology and immunophenotype predict poor outcome in mucinous adenocarcinoma of the uterine cervix. Am J Surg Pathol. 2007;31:664-72.

18. Zhang Y, Liang L, Euscher ED, Liu J, Ramalingam P. Gastric-type mucinous adenocarcinoma of the uterine cervix with neoadjuvant therapy mimicking clear cell carcinoma. Int J Clin Exp Pathol. 2015;8:11798-803. 